\title{
On Liouville Sequences in the Non-Archimedean Case
}

\author{
Hamza Menken ${ }^{1}$ and Abdulkadir Aşan ${ }^{2}$ \\ ${ }^{1}$ Mathematics Department, Science and Arts Faculty, Mersin University, Ciftlikkoy Campus, 33343 Mersin, Turkey \\ ${ }^{2}$ Institute of Science, Mathematics Graduate Program, Mersin University, Ciftlikkoy Campus, 33343 Mersin, Turkey
}

Correspondence should be addressed to Hamza Menken; hmenken@mersin.edu.tr

Received 16 December 2013; Accepted 27 January 2014; Published 5 March 2014

Academic Editors: P. K. Sahoo, H. Wu, and Z. Wu

Copyright (c) $2014 \mathrm{H}$. Menken and A. Aşan. This is an open access article distributed under the Creative Commons Attribution License, which permits unrestricted use, distribution, and reproduction in any medium, provided the original work is properly cited.

We study Liouville numbers in the non-Archimedean case. We deal with the concept of a Liouville sequence in the nonArchimedean case and we give some results both in the $p$-adic numbers field $\mathbb{Q}_{p}$ and the functions field $K\langle x\rangle$.

\section{Introduction}

It is well known that if a complex number $\alpha$ is a root of a nonzero polynomial equation

$$
a_{n} x^{n}+a_{n-1} x^{n-1}+\cdots+a_{1} x+a_{0}=0,
$$

where the $a_{i}$ s are integers (or equivalently, rational numbers) and $\alpha$ satisfies no similar equation of degree $<n$, then $\alpha$ is said to be an algebraic number of degree $n$. A complex number that is not algebraic is said to be transcendental. Liouville's theorem states that, for any algebraic number $\alpha$ with degree $n>1$, there exists $C(\alpha)>0$ such that

$$
\left|\alpha-\frac{a}{b}\right|>\frac{C(\alpha)}{b^{n}}
$$

for all rational numbers $a / b$ with $b>1$. The construction of transcendental numbers has been usually shown using Liouville's theorem. For instance, the transcendence of the number $\xi=\sum_{n=1}^{\infty} 10^{-n !}$ can be easily proved from Liouville's theorem. Also, Liouville's theorem can be applied to prove the transcendence of a large class of real numbers which are called Liouville numbers.

A real number $\xi \in \mathbb{R}$ is called a Liouville number if, for every positive real number $\omega$, there exist integers $a$ and $b(>1)$ such that

$$
\left|\xi-\frac{a}{b}\right|<\frac{1}{b^{\omega}}
$$

It is easy to prove that any real number $\sum_{n=1}^{\infty} a_{n} 10^{-n !}$ with $a_{n} \in$ $\{1,2\}$ is a Liouville number (see $[1,2])$. Real Liouville numbers have many interesting properties and have been investigated by many authors (see [3-8]). In 1975, Erdös [9] proved a very interesting criterion for Liouville series.

Theorem 1 (see Erdös [9]). Let $a_{1}<a_{2}<a_{3}<\cdots$ be an infinite sequence of integers satisfying

$$
\lim _{n \rightarrow \infty} \sup a_{n}^{t^{1 / n}}=\infty
$$

for every $t>0$ and

$$
a_{n}>n^{1+\varepsilon}
$$

for fixed $\varepsilon>0$ and $n>n_{0}(\varepsilon)$. Then,

$$
\alpha=\sum_{n=1}^{\infty} \frac{1}{a_{n}}
$$

is a Liouville number.

Hančl [8] defined the concept of Liouville sequences and generalized the above theorem of Erdös. Now, we recall the definition of Liouville sequences.

Definition 2 (see [8]). Let $\left(a_{n}\right)$ be a sequence of positive real numbers. If, for every $\left(c_{n}\right)$ of positive integers, the sum

$$
\sum_{n=1}^{\infty} \frac{1}{a_{n} c_{n}}
$$


is a Liouville number, then the sequence $\left(a_{n}\right)$ is called a Liouville sequence.

The properties of Liouville sequences were investigated in [8] and some criteria were given for them. In the present work, we define the concept of Liouville sequences in nonArchimedean case and obtain some properties for them.

\section{2. $p$-adic Numbers and $p$-adic Liouville Numbers}

Recall that a norm on a field $K$ is a function $|\cdot|: K \rightarrow[0, \infty)$ satisfying the following conditions:

(i) $|x|=0$ if and only if $x=0$,

(ii) $|x y|=|x||y|$, for all $x, y \in K$,

(iii) $|x+y| \leq|x|+|y|$, for all $x, y \in K$.

A norm on $K$ is called non-Archimedean if it satisfies the extra condition

(iv) $|x+y| \leq \max \{|x|,|y|\}$ for all $x, y \in K$;

otherwise, we say that the norm is Archimedean.

It is well known that the usual absolute value on the rational numbers field $\mathbb{Q}$ (or the real numbers field $\mathbb{R}$ ) is Archimedean. There are interesting non-Archimedean norms. First, we recall the definition of the $p$-adic norm.

Let $p$ be a given prime number. Every nonzero rational number $x$ can be written uniquely under the form

$$
x=p^{v_{p}(x)} \frac{a}{b}
$$

where $v_{p}(x), a, b \in \mathbb{Z}$, and $a$ and $b$ are not divided by $p$. Here, $v_{p}(x)$ is the $p$-adic valuation of $x$. The $p$-adic norm is defined by

$$
|x|_{p}= \begin{cases}p^{-v_{p}(x)}, & x \neq 0 \\ 0, & x=0 .\end{cases}
$$

It is clear that the $p$-adic norm is non-Archimedean. The $p$-adic numbers field $\mathbb{Q}_{p}$ is the completion of the rational numbers field $\mathbb{Q}$ with respect to the $p$-adic norm. Every nonzero $p$-adic number $x \in \mathbb{Q}_{p}$ is uniquely represented in the canonical form

$$
x=p^{m}\left(a_{0}+a_{1} p+a_{2} p^{2}+\cdots\right),
$$

where $m=v_{p}(x), a_{j} \in \mathbb{Z}$, such that $0 \leq a_{j} \leq p-1$ and $a_{0} \neq 0$ $(j=0,1,2, \ldots)$. The unit ball (or the ring of $p$-adic integers) is denoted by $\mathbb{Z}_{p}$ and defined by

$$
\mathbb{Z}_{p}=\left\{x \in \mathbb{Q}_{p}:|x|_{p} \leq 1\right\} .
$$

Similarly, every nonzero $p$-adic integer $x \in \mathbb{Z}_{p}$ is uniquely represented in the canonical form

$$
x=a_{0}+a_{1} p+a_{2} p^{2}+\cdots,
$$

where $a_{j} \in \mathbb{Z}$ and $0 \leq a_{j} \leq p-1(j=0,1,2, \ldots)$. The natural numbers set $\mathbb{N}$ is dense in $\mathbb{Z}_{p}$.

Although the classical Liouville numbers are real numbers that can be rapidly approximated by rational numbers, the $p$-adic Liouville numbers are those numbers that can be rapidly approximated by positive integers in the $p$-adic norm. The $p$-adic Liouville numbers are defined as follows.

Definition 3 (see $[10,11])$. Let $\lambda$ be a $p$-adic integer. If

$$
\lim _{n \rightarrow \infty} \inf \sqrt[n]{|n-\lambda|_{p}}=0,
$$

then $\lambda$ is called a $p$-adic Liouville number.

According to this definition, $\lambda \in \mathbb{Z}_{p}$ is a $p$-adic Liouville number if and only if there exists a sequence of positive integers $a_{n}$ such that

$$
\lim _{n \rightarrow \infty} \sqrt[n]{\left|a_{n}-\lambda\right|_{p}}=0
$$

Example 4. Consider the series $\alpha=\sum_{n=0}^{\infty} p^{n !}$. It is easy to see that the sum is a $p$-adic Liouville number.

The definition above is first introduced by Clark [11] and it is better adapted to differential equations. In fact, consider the differential equation

$$
x f^{\prime}(x)-\lambda f(x)=\frac{1}{1-x}
$$

on a neighborhood $D$ of 0 in $\mathbb{Z}_{p}$, where $\lambda \in \mathbb{Z}_{p} \backslash\{0,1,2, \ldots\}$. This equation has a unique formal solution; namely, $f(x)=$ $\sum_{n=1}^{\infty}(1 /(n-\lambda)) x^{n}$. It is clear that this solution diverges if and only if $\lambda$ is a $p$-adic Liouville number (for details, see [12]). We note that the set of $p$-adic Liouville numbers forms a dense subset of $\mathbb{Z}_{p}$ and every $p$-adic Liouville number is transcendental over $\mathbb{Q}$ (for details, see $[10]$ ).

In general case, the $p$-adic transcendental numbers have been studied by Mahler [13], Adams [14], X. Long Xin [15], Nishioka [16], and others. As a special case, the p-adic Liouville numbers have been studied in [17-21] and others.

\section{Liouville Sequences in the $p$-adic Numbers Fields}

We define the Liouville sequence in $\mathbb{Q}_{p}$ as follows.

Definition 5. Let $\left(a_{n}\right)$ be a sequence of $p$-adic integers. If, for every $\left(c_{n}\right)$ of positive integers, the sum

$$
\sum_{n=1}^{\infty} a_{n} c_{n}
$$

is a $p$-adic Liouville number, then the sequence $\left(a_{n}\right)$ is called a $p$-adic Liouville sequence.

Example 6. Let $p$ be a prime number. It is easy to see that

$$
\left(a_{n}\right)=\left(p^{n !}\right) \subset \mathbb{Z}_{p}
$$

is a $p$-adic Liouville sequence. 
Proof. Let $\left(c_{n}\right) \subset \mathbb{Z}^{+}$be an arbitrary sequence. We want to show that the sum

$$
\gamma=\sum_{n=1}^{\infty} c_{n} p^{n !}
$$

is a $p$-adic Liouville number. Since $\left|c_{n} p^{n !}\right|_{p} \leq\left|p^{n !}\right|_{p}=p^{-n !} \rightarrow$ 0 , the series $\sum_{n=1}^{\infty} c_{n} p^{n !}$ is convergent. We can write

$$
\begin{aligned}
0 & <\left|\gamma-S_{n-1}\right|_{p}^{1 / n}=\left|\sum_{i=0}^{\infty} c_{n+i} p^{(n+i) !}\right|_{p}^{1 / n} \\
& \leq\left[\max \left\{\left|c_{n} p^{n !}\right|_{p},\left|c_{n+1} p^{(n+1) !}\right|_{p}, \ldots\right\}\right]^{1 / n} \\
& =\left[\left|c_{n}\right|_{p}\left|p^{n !}\right|_{p}\right]^{1 / n},
\end{aligned}
$$

where $S_{n}=\sum_{k=1}^{n} c_{k} p^{k !}$. Since $c_{n}$ is an integer, then we get $\left|c_{n}\right|_{p} \leq 1$ and

$$
0<\left|\gamma-S_{n-1}\right|_{p}^{1 / n} \leq\left|p^{n !}\right|_{p}^{1 / n}=p^{-(n-1) !} .
$$

Thus,

$$
\lim _{n \rightarrow \infty}\left|\gamma-S_{n-1}\right|_{p}^{1 / n}=\lim _{n \rightarrow \infty} p^{-(n-1) !}=0 .
$$

This shows that the sum

$$
\gamma=\sum_{n=1}^{\infty} c_{n} p^{n !}
$$

is a $p$-adic Liouville number.

Theorem 7. Let $\left(a_{n}\right)$ be a sequence of positive integers satisfying the following conditions:

$$
v_{p}\left(a_{n}\right)<v_{p}\left(a_{n+1}\right),
$$

for every $n$, and

$$
v_{p}\left(a_{n+1}\right) \geq n^{1+\varepsilon}
$$

for fixed $\varepsilon>0$ and $n>n_{0}(\varepsilon)$. Then, $\left(a_{n}\right)$ is a p-adic Liouville sequence.

Proof. Let $\left(c_{n}\right)$ be an arbitrary sequence of positive integers and let $\varepsilon>0$ be a given arbitrary positive real number. First, we have to prove that the series $\sum_{n=1}^{\infty} a_{n} c_{n}$ is convergent. By condition (24), we know that

$$
v_{p}\left(a_{n+1}\right) \geq n^{1+\varepsilon},
$$

for all $n>n_{0}(\varepsilon)$. It follows from $\left|c_{n}\right|_{p} \leq 1$ that the relation holds

$$
\left|a_{n+1} c_{n+1}\right|_{p} \leq\left|a_{n+1}\right|_{p}=p^{-v_{p}\left(a_{n+1}\right)} \leq p^{-n^{1+\varepsilon}},
$$

for all $n>n_{0}(\varepsilon)$. Thus, $\lim _{n \rightarrow 0} c_{n} a_{n}=0$, so the series $\sum_{n=1}^{\infty} c_{n} a_{n}$ is convergent. By the property $\left|\sum_{n=1}^{\infty} c_{n} a_{n}\right|_{p} \leq \max _{n \in \mathbb{N}}\left|c_{n} a_{n}\right|_{p}$, we obtain that $\alpha=\sum_{n=1}^{\infty} c_{n} a_{n} \in \mathbb{Z}_{p}$. Also, by condition (23), $\alpha \in \mathbb{Z}_{p} \backslash \mathbb{Z}$. Now, we want to show that the sum

$$
\alpha=\sum_{n=1}^{\infty} a_{n} c_{n}
$$

is a $p$-adic Liouville number. Using (23) and (24), we have

$$
\begin{aligned}
0 & <\left|\alpha-S_{n}\right|_{p}^{1 / n}=\left|\sum_{i=1}^{\infty} a_{n+i} c_{n+i}\right|_{p}^{1 / n} \\
& \leq\left[\max \left\{\left|a_{n+1} c_{n+1}\right|_{p},\left|a_{n+2} c_{n+2}\right|_{p}, \ldots\right\}\right]^{1 / n} \\
& =\left|a_{n+1} c_{n+1}\right|_{p}^{1 / n},
\end{aligned}
$$

where $S_{n}=\sum_{k=1}^{n} a_{k} c_{k}$. Since $\left|c_{n+i}\right|_{p} \leq 1$, for all $i=1,2,3, \ldots$, then

$$
\begin{aligned}
0 & <\left|\alpha-S_{n}\right|_{p}^{1 / n}=\left|\sum_{i=1}^{\infty} a_{n+i} c_{n+i}\right|_{p}^{1 / n} \\
& \leq\left|a_{n+1}\right|_{p}^{1 / n}=p^{-v_{p}\left(a_{n+1}\right) / n} \leq p^{-n^{1+\varepsilon} / n} \\
& =p^{-n^{\varepsilon}} \longrightarrow 0 \quad(n \longrightarrow \infty) .
\end{aligned}
$$

Since $S_{n} \in \mathbb{N}$, for all $n$, this shows that $\alpha$ is a $p$-adic Liouville number and the theorem is proved.

Remark 8. Since $v_{p}\left(a_{n}\right) \in \mathbb{N}$, for all $a_{n} \in \mathbb{Z}_{p}$, in Theorem 7 , condition (24) can be replaced by the condition

$$
v_{p}\left(a_{n+1}\right) \geq n^{2} .
$$

In similar way, we can give the following result.

Corollary 9. Let $\left(a_{n}\right)$ be a sequence of p-adic integers satisfying the following conditions:

$$
v_{p}\left(a_{n}\right)<v_{p}\left(a_{n+1}\right),
$$

for everyn, and

$$
v_{p}\left(a_{n+1}\right) \geq n^{2},
$$

for $n>n_{0}$. Then, $\left(a_{n}\right)$ is a p-adic Liouville sequence.

Theorem 10. Let $\left(a_{n}\right)$ be a sequence of positive integers and assume that the relation

$$
0<\left|a_{n}\right|_{p}^{1 / n}<\epsilon
$$

holds for every positive real number $\epsilon>0$ and $n>n_{0}(\varepsilon)$. Then,

(a) $\alpha=\sum_{n=1}^{\infty} a_{n}$ is a $p$-adic Liouville number,

(b) $\left(a_{n}\right)$ is a p-adic Liouville sequence. 
Proof. Let $\epsilon>0$ be a given arbitrary positive real number.

(a) By condition (33), there exists $n_{0}(\varepsilon) \in \mathbb{N}$ such that the relation

$$
0<\left|a_{n}\right|_{p}^{1 / n}<\epsilon
$$

holds, for all $n>n_{0}(\varepsilon)$. Then, we get $\left|a_{n}\right|_{p}<\epsilon^{n}$ and $\left|a_{n}\right|_{p} \rightarrow 0$ as $n \rightarrow \infty$. Hence, the series $\sum_{n=1}^{\infty} a_{n}$ is convergent and by the inequality

$$
\left|\sum_{n=1}^{\infty} a_{n}\right|_{p} \leq \max _{n \in \mathbb{N}}\left|a_{n}\right|_{p} \leq 1,
$$

we have $\alpha=\sum_{n=1}^{\infty} a_{n} \in \mathbb{Z}_{p}$. Now, we show that $\alpha=\sum_{n=1}^{\infty} a_{n}$ is a p-adic Liouville number. Let $\alpha_{n}=\sum_{k=1}^{n} a_{k}$. Then, we can write

$$
0<\left|\alpha-\alpha_{n}\right|_{p}^{1 / n}=\left|\sum_{k=n+1}^{\infty} a_{k}\right|_{p}^{1 / n} \leq \max _{k \geq n+1}\left|a_{k}\right|_{p}^{1 / k}<\epsilon,
$$

for all $n>n_{0}(\varepsilon)$. It follows that

$$
\left|\alpha-\alpha_{n}\right|_{p}^{1 / n} \longrightarrow 0 .
$$

Hence, we obtain that $\alpha=\sum_{n=1}^{\infty} a_{n}$ is a $p$-adic Liouville number.

(b) Let $\left(c_{n}\right)$ be an arbitrary sequence of positive integers. We consider the sum:

$$
\beta=\sum_{n=1}^{\infty} c_{n} a_{n}
$$

We know that the relation

$$
0<\left|a_{n}\right|_{p}^{1 / n}<\epsilon
$$

holds, for all $n>n_{0}(\varepsilon)$. Since $\left|c_{n}\right|_{p} \leq 1$, we get

$$
\left|a_{n} c_{n}\right|_{p} \leq\left|a_{n}\right|_{p}<\epsilon^{n}
$$

and $\left|a_{n} c_{n}\right|_{p} \rightarrow 0$ as $n \rightarrow \infty$. Hence, the series $\sum_{n=1}^{\infty} a_{n} c_{n}$ is convergent and by the inequality

$$
\left|\sum_{n=1}^{\infty} a_{n} c_{n}\right|_{p} \leq \max _{n \in \mathbb{N}}\left|a_{n} c_{n}\right|_{p} \leq 1,
$$

we have $\beta=\sum_{n=1}^{\infty} a_{n} c_{n} \in \mathbb{Z}_{p}$. Let $\beta_{n}=\sum_{k=1}^{n} c_{k} a_{k}$. Then, we can write

$$
\begin{aligned}
0 & <\left|\beta-\beta_{n}\right|_{p}^{1 / n}=\left|\sum_{k=n+1}^{\infty} c_{k} a_{k}\right|_{p}^{1 / n} \\
& \leq \max _{k \geq n+1}\left|c_{k} a_{k}\right|_{p}^{1 / n} \leq \max _{k \geq n+1}\left|a_{k}\right|_{p}^{1 / n}<\epsilon
\end{aligned}
$$

for all $n>n_{0}(\varepsilon)$. It follows that

$$
\left|\beta-\beta_{n}\right|_{p}^{1 / n} \longrightarrow 0
$$

Since $\beta_{n} \in \mathbb{N}$, for all $n$, we obtain that $\beta=\sum_{n=1}^{\infty} c_{n} a_{n}$ is a $p$-adic Liouville number. So, the theorem is proved.

\section{The Liouville Sequences in the Functions Field}

Let $K$ be an arbitrary field, $x$ an indeterminate, $K[x]$ the ring of all polynomials in $x$ with coefficients in $K, K(x)$ the field of all rational functions in $x$ with coefficients in $K$, and $K\langle x\rangle$ the field of all formal series

$$
z=a_{k} x^{k}+a_{k-1} x^{k-1}+a_{k-2} x^{k-2}+\cdots
$$

in $x$, where the coefficients $a_{k}, a_{k-1}, a_{k-2}, \ldots$ are in $K$. Thus, $K(x)$ is the quotient field of $K[x]$ and a subfield of $K\langle x\rangle$.

A valuation $|z|$ in $K\langle x\rangle$ is now defined by putting $|0|=0$ and $|z|=e^{k}$ if

$$
z=a_{k} x^{k}+a_{k-1} x^{k-1}+a_{k-2} x^{k-2}+\cdots
$$

and $a_{k} \neq 0$.

If $z$ lies in $K[x]$, then $\log |z|=\operatorname{deg} z$.

It is clear that this norm is a non-Archimedean and so $K\langle x\rangle$ is a non-Archimedean field with this norm.

The analogue of Liouville's theorem states that if $\alpha \in K\langle x\rangle$ is an algebraic number of degree $n \geq 2$ over $K(x)$, then there exists a positive constant $C(\alpha)$ depending only on $\alpha$ such that

$$
\left|\alpha-\frac{a}{b}\right| \geq \frac{C(\xi)}{b^{n}}
$$

for all $a, b \in K[x](b \neq 0)$ (see [22]). Some results on the Liouville numbers in the functions field were obtained in [20]. Now, we recall the definition of a Liouville number in this field.

Definition 11. An element $\xi \in K\langle x\rangle$ is called a Liouville number if, for every $\omega \in \mathbb{R}^{+}$, there existed integer $a, b \in$ $K[x] \backslash\{0\}$ with $|b|>1$ such that

$$
0<\left|\xi-\frac{a}{b}\right|<\frac{1}{b^{\omega}} \text {. }
$$

We define the concept of a Liouville sequence in the function fields as follows.

Definition 12. Let $\left(z_{n}\right) \subset K\langle x\rangle$. If, for every $\left(a_{n}\right) \in K[x]$, the sum

$$
\sum_{n=1}^{\infty} \frac{1}{a_{n} z_{n}}
$$

is a Liouville number, then the sequence $\left(z_{n}\right)$ is called a Liouville sequence.

Theorem 13. Let $\left(z_{n}\right) \subset K\langle x\rangle$ satisfying the following conditions:

$$
0<\operatorname{deg}\left(z_{n}\right)<\operatorname{deg}\left(z_{n+1}\right)
$$

for every $n$, and

$$
\operatorname{deg}\left(z_{n+1}\right)>n^{1+\varepsilon} \text {, }
$$

for fixed $\varepsilon>0$ and $n>n_{0}(\varepsilon)$. Then, $\left(z_{n}\right)$ is a Liouville sequence. 
Proof. Let $\left(a_{n}\right) \subset K[x]$ be an arbitrary sequence and $\varepsilon>$ 0 be an arbitrary positive real number. First, we show that $\sum_{n=1}^{\infty}\left(1 / a_{n} z_{n}\right)$ is convergent in $K\langle x\rangle$. From condition (50), we have

$$
\left|\frac{1}{a_{n} z_{n}}\right|=e^{-\operatorname{deg}\left(a_{n} z_{n}\right)} \leq e^{-\operatorname{deg}\left(z_{n}\right)}<e^{-(n-1)^{1+\varepsilon}}
$$

for all $n>n_{0}(\varepsilon)$. Then, we get $1 / a_{n} z_{n} \rightarrow 0$. Thus, the series $\sum_{n=1}^{\infty}\left(1 / a_{n} z_{n}\right)$ is convergent. Now, we want to show that the sum

$$
\alpha=\sum_{n=1}^{\infty} \frac{1}{a_{n} z_{n}}
$$

is a Liouville number. Let $S_{n}=\sum_{k=1}^{n}\left(1 / a_{k} z_{k}\right)$. Then, we write

$$
0<\left|\alpha-S_{n}\right|^{1 / n}=\left|\sum_{i=1}^{\infty} \frac{1}{a_{n+i} z_{n+i}}\right|^{1 / n} \leq\left[\max _{k \geq n+1} \frac{1}{\left|a_{k} z_{k}\right|}\right]^{1 / n} .
$$

Since $\operatorname{deg}\left(a_{k}\right) \geq 0$, we obtain that

$$
\begin{aligned}
{\left[\max _{k \geq n+1} \frac{1}{\left|a_{k} z_{k}\right|}\right]^{1 / n} } & =\left[\max _{k \geq n+1} e^{-\operatorname{deg}\left(a_{k}\right)-\operatorname{deg}\left(z_{k}\right)}\right]^{1 / n} \\
& \leq\left[\max _{k \geq n+1} e^{-\operatorname{deg}\left(z_{k}\right)}\right]^{1 / n} .
\end{aligned}
$$

By (49), we can write

$$
\max _{k \geq n+1} e^{-\operatorname{deg}\left(z_{k}\right)}=e^{-\operatorname{deg}\left(z_{n+1}\right)}
$$

and by using (50) we get

$$
\begin{aligned}
{\left[\max _{k \geq n+1} \frac{1}{\left|a_{k} z_{k}\right|}\right]^{1 / n} } & \leq\left[\max _{k \geq n+1} e^{-\operatorname{deg}\left(z_{k}\right)}\right]^{1 / n} \\
& =e^{-\operatorname{deg}\left(z_{n+1}\right) / n} \leq e^{-n^{1+\varepsilon} / n} \\
& =e^{-n^{\varepsilon}} \longrightarrow 0 \quad(n \longrightarrow \infty)
\end{aligned}
$$

This shows that $\left|\alpha-S_{n}\right|^{1 / n} \rightarrow 0, n \rightarrow \infty$. Also, by condition (49), $z_{n} \in K[x]$ and so $S_{n} \in K(x)$. Thus, we prove that $\alpha$ is a Liouville number.

\section{Conflict of Interests}

The authors declare that there is no conflict of interests regarding the publication of this paper.

\section{Acknowledgments}

This work is supported by Mersin University. The authors would like to thank the editors and reviewers for their useful suggestions.

\section{References}

[1] A. Baker, Number Theory, Cambridge University Press, Cambridge, UK, 1975.

[2] Y. Bugeaud, Approximation by Algebraic Numbers, Cambridge University Press, Cambridge, UK, 2004.

[3] K. Mahler, "Zur Approximation der Exponentialfunktion und des Logarithmus I, II," Journal für die Reine und Angewandte Mathematik, vol. 166, pp. 118-150, 1932.

[4] J. F. Koksma, "Über die Mahlersche Klasseneinteilung der transzendenten Zahlen und die Approximation komplexer Zahlen durch algebraische Zahlen," Monatshefte für Mathematik und Physik, vol. 48, no. 1, pp. 176-189, 1939.

[5] P. Erdös, "Representation of real numbers as sums and products of Liouville numbers," Michigan Mathematical Journal, vol. 9, pp. 59-60, 1962.

[6] W. J. Leveque, “On Mahler's U-Numbers," London Mathematical Society, pp. 220-229, 1953.

[7] K. Alniaçik, "On Um-numbers," Proceedings of the American Mathematical Society, vol. 85, no. 4, pp. 499-505, 1982.

[8] J. Hančl, "Liouville sequences," Nagoya Mathematical Journal, vol. 172, pp. 173-187, 2003.

[9] P. Erdös, "Some problems and results on the irrationality of the sum of infinite series," Michigan Mathematical Journal, pp. 1-7, 1975.

[10] W. H. Schikhof, Ultrametric Calculus, Cambridge University press, Cambridge, UK, 2006.

[11] D. Clark, "A note on the $p$-adic convergence of the solutions of linear differential equations," Proceedings of the American Mathematical Society, vol. 17, pp. 262-269, 1966.

[12] M. van der Put and L. Taelman, "Local p-adic differential equations, $p$-Adic Mathematical Physics," in AIP Conference Proceedings, vol. 826, pp. 291-297, The American Institute of Physics, Melville, NY, USA, 2006.

[13] K. Mahler, "Uber eine Klassen-Einteilung der p-adischen Zahlen," Mathematica, vol. 3, pp. 177-185, 1935.

[14] W. W. Adams, "Transcendental numbers in the p-adic domain," The American Journal of Mathematics, vol. 88, pp. 279-308, 1966.

[15] X. Long Xin, "Mahler's Classification of $p$-adic numbers," Pure and Applied Mathematics, vol. 5, pp. 73-780, 1989.

[16] K. Nishioka, "p-adic transcendental numbers," Proceedings of the American Mathematical Society, vol. 108, no. 1, pp. 39-41, 1990.

[17] H. Menken, "An investigation on p-adic U-number," Istanbul Universitesi Fen Fakültesi Matematik Dergisi, vol. 59, pp. 111-143, 2001.

[18] V. V. Beresnevich, V. I. Bernik, and E. I. Kovalevskaya, "On approximation of $p$-adic numbers by $p$-adic algebraic numbers," Journal of Number Theory, vol. 111, no. 1, pp. 33-56, 2005.

[19] H. Menken and K. R. Mamedov, "Point on curves whose coordinates are $p$-adic $U$-numbers," in Proceedings of the 2nd International Conference on $p$-Adic Mathematical Physics, vol. 826 of AIP Conference Proceedings, pp. 267-273, Melville, NY, USA, September 2006. 
[20] T. Chaichana, T. Komatsu, and V. Laohakosol, "Liouville numbers in the non-archimedean case," Publicationes Mathematicae, vol. 77, no. 1-2, pp. 39-63, 2010.

[21] H. Menken and A. Aşan, "On some properties of Liouville numbers in the non-Archimedean case," European Journal of Pure and Applied Mathematics, vol. 6, no. 2, pp. 239-246, 2013.

[22] K. Mahler, "On a theorem of Liouville in fields of positive characteristic," Canadian Journal of Mathematics, vol. 1, pp. 397400, 1949. 


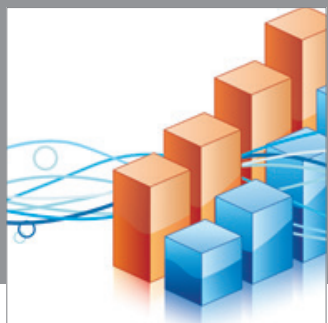

Advances in

Operations Research

mansans

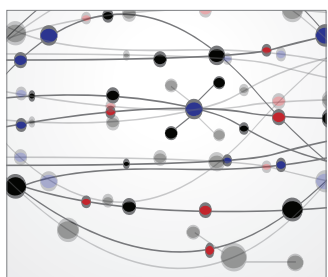

The Scientific World Journal
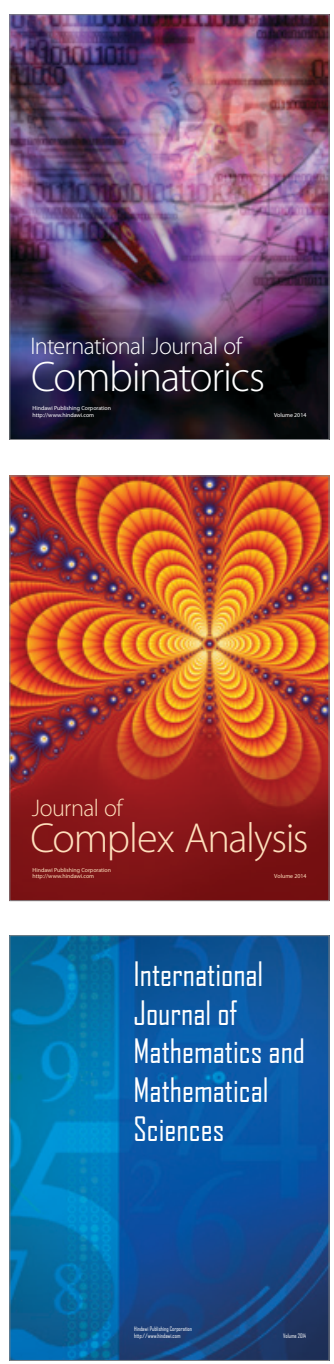
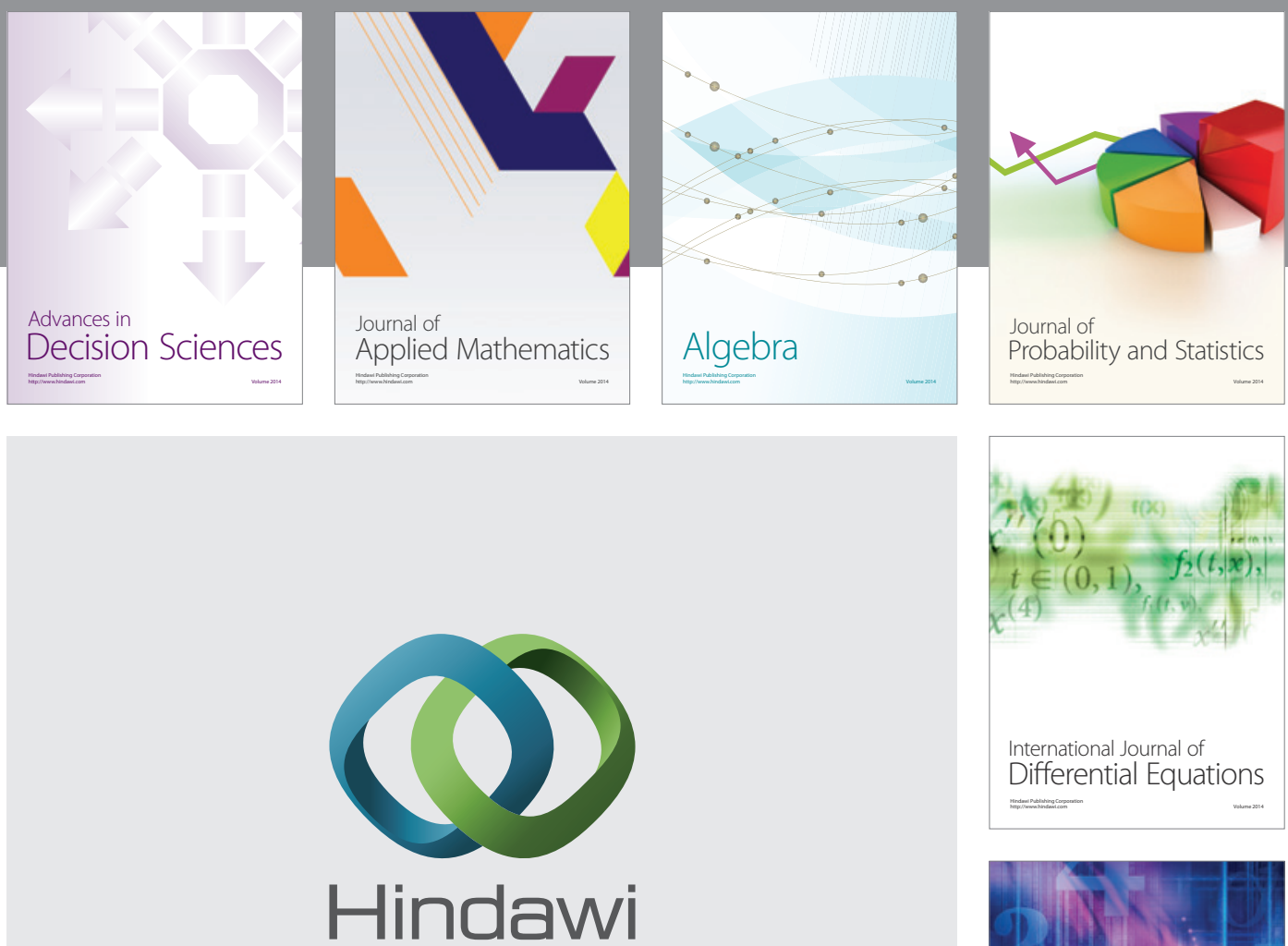

Submit your manuscripts at http://www.hindawi.com
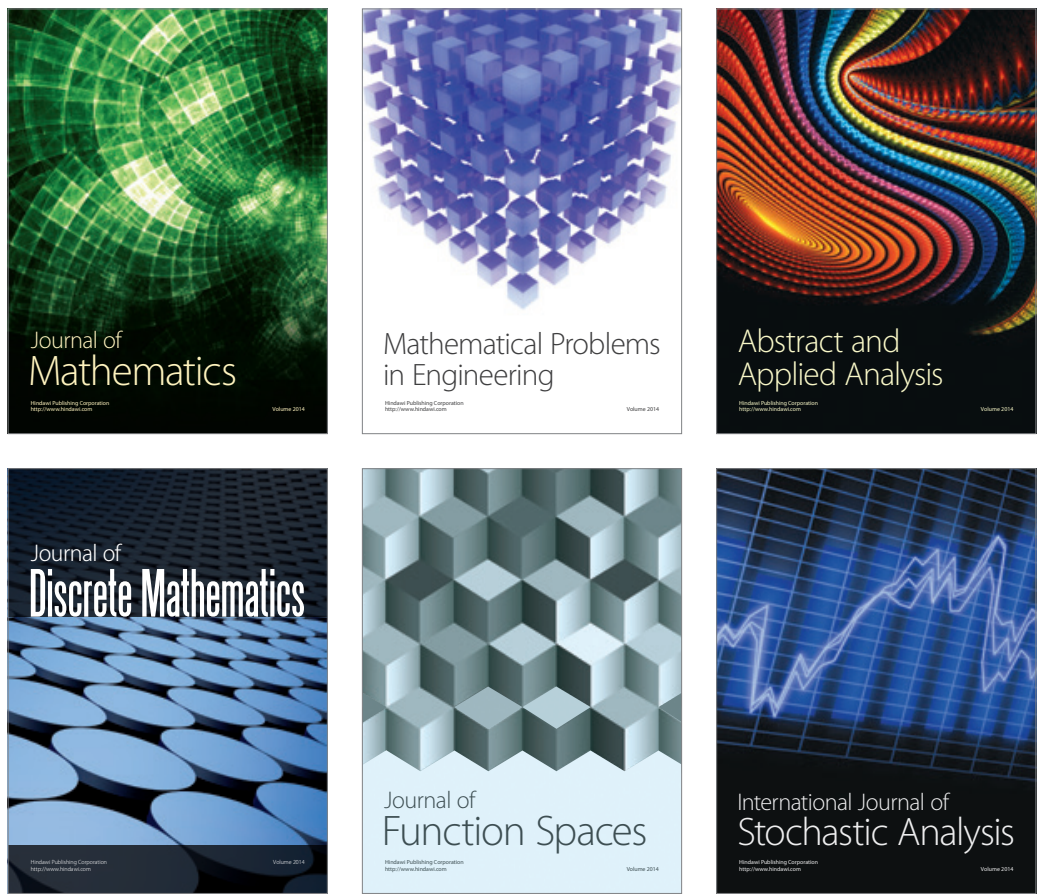

Journal of

Function Spaces

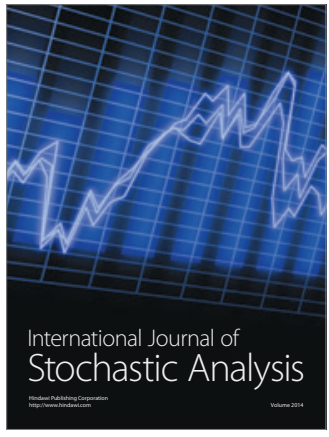

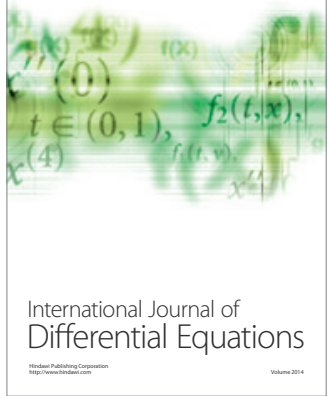
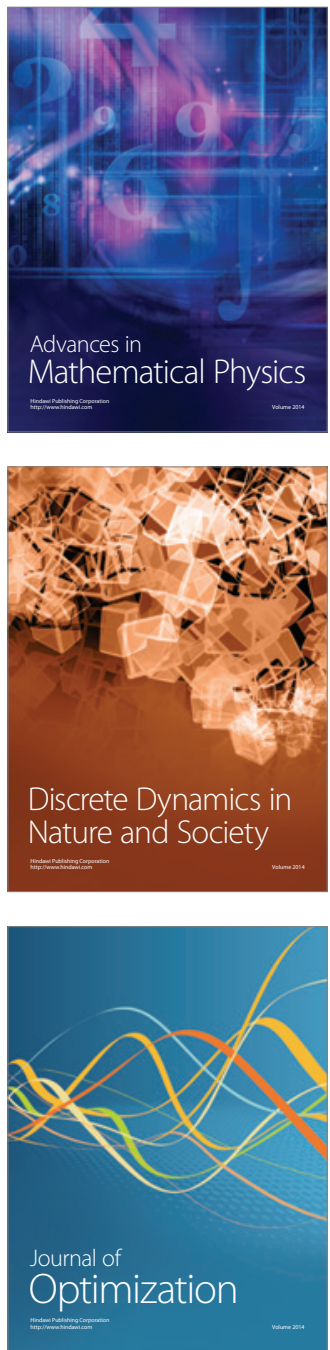